C2006 IEEE. Personal use of this material is permitted. However, permission to reprint/republish this material for advertising or promotional purposes or for creating new collective works for resale or redistribution to servers or lists, or to reuse any copyrighted component of this work in other works must be obtained from the IEEE. 


\title{
Applications of RFID in Pharmaceutical Industry
}

\author{
Manohar Potdar ${ }^{1}$ Elizabeth Chang ${ }^{2}$ Vidyasagar Potdar ${ }^{2}$ \\ ${ }^{1}$ Department of Pharmaceutical Quality Assurance, Poona College of Pharmacy, Bharati Vidyapeeth University, Pune, India \\ potdar_manohar@yahoo.com,www.drmanoharpotdar.com \\ ${ }^{2}$ Digital Ecosystems and Business Intelligence Institute, Curtin Business School, Curtin University of Technology, Australia \\ \{Elizabeth.Chang, Vidyasagar.Potdar\}@cbs.curtin.edu.au www.debii.curtin.edu.au www.rfidtamperdetection.com
}

\begin{abstract}
Security and safety are two important features desired in pharmaceutical supply chain and achieving the same is a challenging task. The need to secure and authenticate pharmaceutical products has increased tremendously with the emerging counterfeit product market. The motivation to introduce counterfeit pharmaceutical products in the supply chain could be to gain rapid economic benefits or affecting the reputation of strong brand in the pharmaceutical industry. RFID technology can be used to deter counterfeiting attempts. It can also be used in various other domains in the pharmaceutical industry. The main aim of this paper is to outline all the applications of RFID in the pharmaceutical industry. After explaining the main applications, we discuss how information hiding techniques could be used with RFID to offer efficient expiry date management, pharmaceutical tamper detection, and fraud detection and prevention.
\end{abstract}

\section{INTRODUCTION}

Pharmaceutical industry is one of the major industries, which deals directly with the healthcare of human beings and animals. The main activities in this industry are two, namely, manufacturing of medicines and distribution of manufactured medicines to the public. Since there is no "seconds" concept in the pharmaceutical industry, like in many other industries e.g. foot wear, garment etc. where goods with slight defects may also be sold legally, at slightly reduced price, however, defective or sub standard medicines cannot be sold in the market. Hence all medicines sold must meet certain universally accepted quality parameters. These parameters or characteristic are as follows: Identity, Strength, Safety, and Purity. Every medicinal product must meet these requirements, since these parameters will provide the desired efficacy in the final finished pharmaceutical products.

Out of these four parameters, 'IDENTITY' is the most important characteristic of the pharmaceutical product. Identity refers to correct and timely identification of product, people, manufacturing activities, materials, machines, equipment, manufacturing processes and location etc., where the manufacturing activities are carried out. In addition to this, proper identification of vendor; from which various materials are purchased and the distribution agent and its location are also important. This not only helps the manufacturer and the distributor of the medicinal product but the Department of Foods and Drugs Administration (FDA) requires it as well.

RFID (Radio Frequency Identification) is a very valuable business and technology tool in this regard, as it holds the promise of replacing existing identification technologies like bar code. RFID offers strategic advantages for businesses because it can track inventory in the supply chain more efficiently, provide real-time in transit visibility (ITV) and monitor general enterprises assets.

Proper identification of various materials; like raw materials, packaging materials, finished pharmaceutical product etc. is one of the prime requirement of quality assurance in pharmaceutical industry. This identification is required at every stage and every location in a pharmaceutical manufacturing and distribution operations, and RFID can here become a solution of choice, to improve the business performance.

In this paper we discuss the potential applications of RFID technology in pharmaceutical industry, which not only includes inventory and warehouse management but also other areas like access control and theft control etc.

However before beginning a detailed description on the major applications we first explain the basic RFID technology. The paper is organized as follows. In Section 2, we explain the basics of RFID technology. In Section 3, we discuss the current state of art of the Indian pharmaceutical industry. In Section 4, we outline all major applications of RFID in the pharmaceutical industry. In Section 5, we describe how information hiding can add new dimensions to RFID technology and finally, we conclude the paper in Section 6.

\section{Preliminary Knowledge}

In this section we discuss the basics of RFID architecture which includes the RFID tags and its internal data structure, RFID readers, RFID middleware, backend legacy systems like SCM, ERP, etc.

\section{A. RFID Basics}

RFID system is composed of three main elements i.e. the RFID Tag which contains data that uniquely identifies an object, a RFID Reader which reads and writes the data on the tags and finally, a backend database which is used to record the data collected by the tag readers [8].

Every RFID Tag, contains a small microchip which has limited computing capabilities limited to simple logical operations and is also used for storage. The storage can be read only, write only or any other combination. The tag also comprises of a small antenna which can infer the radio waves.

The RFID Reader, send radio waves to the RFID tags, to enquire about their data contents. The tags then respond by 


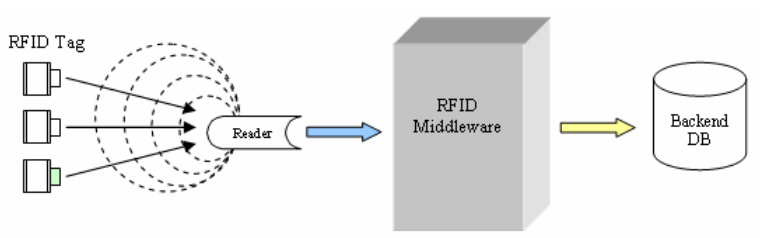

Fig. 1 RFID Architecture

sending back the requested data. The readers may have some processing and storage capabilities. The reader is linked via the RFID middleware with the backend database, to do any other computationally intensive data processing.

The RFID middleware, is designed to provide the messaging, routing, and connectivity for reliable data integration with existing backend systems such as Enterprise Resource Planning (ERP) Systems and Warehouse Management Systems (WMS), etc [2].

Finally, the backend database, stores the complete record of RFID items. It maintains the detailed item information as well as tag data, which shall be consistent with the one read from the RFID.

The low end (or cheap) RFID tags are passive in nature i.e. they only get activated in presence of the reader's radio waves. However some next generation tags are equipped with battery and hence are active in nature. This nature of the tags is useful in deciding the range in which they can be accessed. Active tags have a higher range compared to passive tags. If passive tags need a higher range, the reader should be powerful enough and posses high range. Fig. 1 depicts the relationship between RFID Tag, RIFD Reader, RFID Middleware, and Backend Database.

\section{B. RFID Tag Data Structure}

Electronic Product Codes (EPC) defines the RFID tag data structure. It allows unique identification of manufacturer, its products and individual items belonging to that product. The inbuilt data structure of EPC is now discussed for an in depth analysis. EPC was first developed by Auto-ID Center in MIT in 1999. This centre developed the initial RFID standard and later transferred to EPCGlobal for commercialization in late 2003. EPC proposed two data structures of the RFID, firstly a 64 bit EPC was designed primarily for testing, and 96 bit EPC was designed for commercialization. According to [9], 96 bit chip would be the dominant data format for commercial RFID tags. The data structure of EPC within the RFID is shown in Table 1 and 2.

TABLE 1

DATA STRUCTURE FOR EPC-64

\begin{tabular}{|l|c|c|c|c|}
\hline Element & Header & EPCManager & Object Class & Serial Number \\
\hline Bits & $\mathbf{2}$ & $\mathbf{2 8}$ & $\mathbf{2 4}$ & $\mathbf{1 0}$ \\
\hline Value $_{10}$ & $\mathbf{0 - 3}$ & $\mathbf{0 - 2 6 8 4 3 5 4 5 5}$ & $\mathbf{0 - 1 6 7 7 7 2 1 5}$ & $\mathbf{0 - 1 0 2 3}$ \\
\hline Example & & Toyota & Camry & $\mathbf{1 A U T 3 1 5}$ \\
\hline
\end{tabular}

TABLE 2

DATA STRUCTURE FOR EPC-96

\begin{tabular}{|l|c|c|c|c|}
\hline Element & Header & EPCManager & Object Class & Serial Number \\
\hline Bits & $\mathbf{8}$ & $\mathbf{2 8}$ & $\mathbf{2 4}$ & $\mathbf{3 6}$ \\
\hline Value $_{10}$ & $\mathbf{0 - 3}$ & $\mathbf{0 - 2 6 8 4 3 5 4 5 5}$ & $\mathbf{0 - 1 6 7 7 7 2 1 5}$ & $\mathbf{0 - 6 8 7 1 9 4 7 6 7 3 5}$ \\
\hline Example & & Toyota & Camry & 1AUT315 \\
\hline
\end{tabular}

The EPC is partitioned into four sections as shown in Table 1 and 2. The first partition is the Header that determines, which EAN.UCC key is used and how many bits are allocated to the remaining sections. The second partition is the EPC Manager which identifies the product manufacturer e.g. Toyota. The third partition is the Object Class which is a unique identifier for the product manufactured by the manufacturer e.g. Camry. This value is defined by the product manufacturer, although it might follow some industry standards. Finally, the last partition is the Serial Number which is assigned to each item belonging to a class of product e.g. registration number of the Toyota Camry i.e. 1AUT315.

\section{RFID Middleware}

RFID Middleware locates itself between the RFID reader and the backend database systems. It works within the organization, moving information (i.e. EPC) from the RFID tag to the integration point of high-level supply-chain management systems, through a series of data related services. In general, the RFID middleware [2], manages the readers and extracts EPC data from the readers, followed by tag data filtering, aggregating, and counting, and finally sending the data to the enterprise WMS, and the backend database.

From the architectural perspective, RFID middleware has three layers of functionality: Reader API, Data Management, and Integration Management. This is shown in Fig. 2 [2]. The reader API provides the interface, which can be used by the middleware to interact with the reader. Meanwhile, it also supports flexible interaction patterns e.g. asynchronous subscription and active strategies to sense the reader. The data management layer mainly deals with filtering redundant data, aggregating duplicate data, and routing data to appropriate destination based on the content. The integration layer provides data connectivity to legacy data source and supporting systems at different integration level and thus can be further divided into three sub-layers: application integration, partner integration, and process integration.

The middleware communicates with the web broker, the backend database and the WMS or ERP. The Web Broker is employed to share, query, and update the public data structure and schema of RFID tag data, by exchanging XML documents across trading partners, in a web-based logistics network. Any update of the data structure will be reflected and propagate to all involved RFID data items stored in the backend database. From the standardization view, it enables users to exchange RFID-related data with trading partners through the Internet. From the implementation angle, it is a virtual web services consumer and provider running as peers in the distributed logistics network. 


\section{INDIAN PHARMACEUTICAL INDUSTRY - CURRENT STATE OF THE ART}

In the Indian pharmaceutical industry, presently, RFID technology is practically nonexistent. If at all, only a very small parentage of industry may be using it, and that too in a very limited area of activities. The entire materials management and inventory control tracking is done by using computerized systems; however, data collection is largely manual.

Any sizable pharmaceutical manufacturing unit ${ }^{1}$ handles at least 500 different kinds of raw material and equally high number of primary and secondary packing materials. Finished product and their various packs can vary from 200 to 400, and this shows there is ample scope for thinking of implementation RFID technology in this industry.

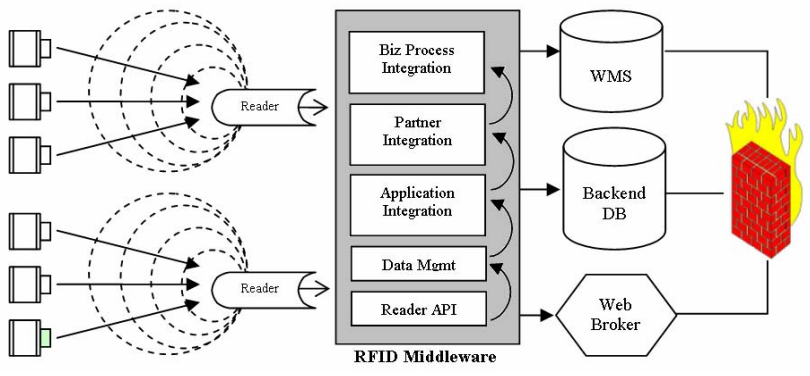

Fig. 2 Generic RFID Middleware Architecture

The other areas of application are the sales and distribution of the pharmaceutical product. Generally there is a chain of distribution starting from manufacturing units (e.g. factory), to company's warehouse, main distributor having depots, wholesalers, and finally a chain of retail pharmacies.

The movement of the product from the factory to the retail pharmacy is a long chain. Correct and timely information about the stock movement and inventory at every stage is very important. Since at present the stock taking is practically manual and communication through electronic mails, this is definitely time and effort consuming and hence, there is a lot of scope for adopting RFID in this area.

The other major problem faced by the pharmaceutical industry is counterfeit drugs and drug diversion because of different pricing and taxation structures prevailing in the country.

Other specific areas where application of RFID technology can be thought of is access control, theft control; materials identification tracking, asset tracking etc. Currently all the above areas are highly vulnerable and no good controls are available; hence RFID seems to be the best alternative.

\section{RFID APPLICATIONS IN THE PHARMACEUTICAL INDUSTRY}

FDA departments all over the world are interested in knowing the correct identification of medicinal product,

\footnotetext{
${ }^{1}$ Here unit refers to a pharmaceutical company, where medicines are manufactured
}

materials used in the preparation of medicinal products, equipments and the areas used for manufacturing etc. They require and demand documentary evidence for all these parameters; however at present they are not insisting on a particular technology to facilitate this process. RFID can be a good candidate to address this problem. We are sure the FDA departments will soon begin to enforce the use of this technology in manufacturing and distribution system of pharmaceutical product. Now let us discuss some of the major areas of application of RFID technology in pharmaceutical industry.

\section{A. Inventory and Warehousing Management}

The pharmaceutical industry handles different types of materials and hence, identity and stock management is a very crucial and important activity. The materials handled are classified in different categories and their identification and movement tracking is very essential for the management of the pharmaceutical unit and also a critical requirement of the FDA in every country. Tracking of all such material is a big task and a challenge that RFID technology can address. There are two main areas in inventory management and control where RFID can be useful, firstly, tracking entry and exit of raw materials and finished goods, and secondly, smart shelving and smart search $[1,7]$.

\section{Entry and Exit Tracking}

RFID technology can have direct and useful applications in tracking the entry and exit of goods in a warehouse. All material entering the pharmaceutical manufacturing unit must be identified by the supplier name, manufacturer name, product name, batch numbers, quantity, dates of manufacturing and expiry etc. Other than that, there is a list of certified vendors, from which a particular material must be purchased. If each arriving container has a RFID tag to identify the suppliers or manufacturers, it would facilitate easier track and trace, at the same time it would help to certify that the material has arrived from the approved vendor. However, if this checks fails then the material can be quarantined for further investigation. This test can be done at the entry site of the materials department or warehouse. Once the raw material is received and approved, it goes on changing its status in the warehouse and in the manufacturing departments, as shown in Table 3.

TABLE 3

DIFFERENT STATES OF RAW MATERIAL

\begin{tabular}{|l|l|}
\hline Material received & Material approved for use. \\
\hline Material sampled & $\begin{array}{l}\text { Material not approved for use or } \\
\text { required. }\end{array}$ \\
\hline Material under test & $\begin{array}{l}\text { Material dispensed for a particular } \\
\text { product and batch etc. }\end{array}$ \\
\hline
\end{tabular}

RFID tags could be assigned to the material after at each of the status shown in Table 3 . This would prevent accidental use of the wrong category of materials, since FDA insist on use of 
tested and approved materials in manufacturing. RFID tags can help to validate the manufacturing and maintain quality control and quality assurance by documenting that only approved materials as being used.

If a central data recording system is used in the plant to record all the dispensed materials could be easily identified, recorded and inventory status maintained automatically in the plant, which would further help in identify reorder level by the purchase department.

If RFID tags are fixed on transportable finished goods packs, it would facilitate the maintenance of automatic track and trace of all the materials leaving the manufacturing unit, which would further help in knowing and maintaining correct stock of all the finished product goods $[1,7]$.

\section{Smart Shelf and Smart Search}

Smart shelves and smart search is a very new concept, and it would be while till it makes inroads in the Indian pharmaceutical industry. Smart shelves in simple words could be explained as 'shelves that know what they carry', which means, they can be searched from a distant location and can offer the precise location of product or palette. The capability to search the palette location within the warehouse would save time and reduce the manpower associated with such administrative tasks, as well as facilitate decision making. In the information age, where capability to search is deemed as power, providing this feature to the warehouse shelves would definitely be a major competitive advantage. RFID technology can offer smart shelving and searching solutions. To do this all shelves in a warehouse should be first uniquely identified; this can be done using RFID tags. Each unique shelf location could then be used to associate it with a palette. Hence, whenever a new consignment arrives at the warehouse, the RFID system can direct the consignment to the most appropriate location within the warehouse. Since the RFID system is capable to keep track of all the empty shelf space in the warehouse, the whole process can be automated. The whole warehouse can thus be operated from a remote location. The concept of smart search can further be extended to facilitate manual lookup, e.g. in large warehouses, it would be extremely beneficial if the exact location is highlighted by a Light Emitting Device (LED) or a bulb. Hence when a palette is searched from a computing device the relevant LED would light up to exactly show the location of the desired palette. This would facilitate the warehouse operator to load and unload palettes when they enter or exit the warehouse.

\section{B. Access Control}

In pharmaceutical manufacturing operations many activities, areas, materials and documents are required to be handled by several people. Some of these are critical and confidential and hence not everyone is allowed to access them. This is required to maintain a level of secrecy and operational safety. One approach to address this issue is providing selective access. RFID technology may be used here to restrict access to authorized resources. We now discuss some areas in the pharmaceutical industry, where RFID can be used for access control $[1,7]$.

\section{Sterile Product Processing}

'Sterile Products' are those products, which are totally free from micro organisms. This area is maintained under strict vigilance and is identified as Class $\mathrm{A}$ area in pharmaceutical manufacturing units. Only those people who have been certified and approved by the System Suitability Test (SST) are allowed to enter this area. The entry must be strictly restricted to other people who have not been certified by SST. RFID tags could be issued to SST certified employees to access these premises.

\section{Critical Process Areas}

Pharmaceutical manufacturing processes are divided in two categories - critical processes and non-critical processes. Critical processes are those where some important step of the process is carried out e.g. addition of active drug materials. Here it is the duty of the supervising staff to ensure that correctly identified material, in correct quantity is added by correctly defined method. At the same time, documentary evidence of following all these precautionary measures should be recorded and preserved. RFID solution can be used for access control in this scenario as well. The access control can not only be deployed for humans but for the materials as well. RFID tags could be added to raw materials to ensure that unacceptable raw materials do not enter restricted premises.

\section{Documentation Room}

All pharmaceutical processes are required to be validated before they are used to manufacture a specific product. Process validation is defined by US-FDA. as follows "Process validation is establishing documented evidence which provide a high degree of assurance that a specific process (such as the manufacture of pharmaceutical dosage forms) will consistently produce a product meeting its predetermined specifications and quality characteristics" [10].

This shows that documentation plays a very important role in the pharmaceutical industry. Some examples of confidential documents include reports such as product development data, product stability data, technical audit report of FDA, product recall and market complaints etc. Documents like these and several others are required to be handled with utmost secrecy and confidentiality. These documents should be protected from being misused and intentionally corrupted. All critical documents must be clearly identifiable as original and genuine. Such documents have a separate physical area having restricted physical entry and selective access. RFID can be used for access control in two ways, firstly to restrict human access to high security areas like this, and secondly to prevent movement of such document in and out from these premises. All critical and confidential documents could be printed on RFID tagged papers. RFID tagged paper is similar to a normal paper, but has 
a barcode and a RFID chip underneath. It can be printed from a RFID enabled printer. Hence using RFID can address the security issue with physical movement of confidential documents.

\section{Entry and Exit to Building}

Entry and exit from a building can be controlled using RFID. If each employee is given a RFID tag as an Identification Card, the system would only allow authorized personal to enter or exit high security facilities.

\section{Machine Operation Access}

In pharmaceutical industry there are many fully automatic machines working on programmable logic control (PLC). In this case there are two major issues, which need to be addressed, firstly, only the fully trained personnel should operate these machines i.e. no other person should have access to operate these machines and secondly, fully trained personnel should not be allowed to change the PLC without proper authorization. This is because, if some critical parameters are changed it could have adverse effect on the product quality and standard. Same examples of the parameters include sterilization time, steam pressure and temperature, rotation per minute of a mixer, times of mixing operation etc. RFID can be used in controlling access to such critical machine operation.

\section{Anti-Theft}

Theft is one of the major issues faced by almost all pharmaceutical companies. The problem arises right from the project stage i.e. construction of pharmaceutical manufacturing plant to the operation of a fully equipped and running manufacturing unit. The thefts can be of different nature e.g. costly machine parts, electric motors, costly drugs, pharmaceuticals, poisonous and highly toxic substances or important confidential documents. Now let us see how RFID technology can help us in preventing and tracing the thefts of this nature.

Costly spares and machine parts, which are classified as Class-A or Class-X (i.e. critical and costly parts) can be tagged with RFID tags so that their movement and inventory can be monitored at all times. The ability to permanently affix a tag to such high cost items in a location and know exactly where the item is at all times is something no other technology can offer.

RFID can be used to track and trace valuable assets for example, triggering an alarm to sound or a camera to take a photo when tagged items passes through doorways. This will have an improved control over the theft of such item from the plant [7].

On the similar lines other costly materials can also be controlled. RFID tags could be placed in dry powder material stored in bulk containers, to control the theft by tracing the movement of such containers from its place of storage. In this fashion we may be able to control the theft of material like costly products, costly drugs, poisonous and highly toxic substances.

\section{Asset Management}

In pharmaceutical industry RFID can also be used to track different assets like furniture and fixtures, equipments, analytical instruments, computers and computer accessories etc. If all such items are RFID tagged then it would facilitate automated inventory audit as well as movement of such items. Even interdepartmental movements could be tracked.

\section{E. Item Level Tagging}

If RFID tags become cost effective then item level tagging could be brought in place. A finished pharmaceutical pack containing 100 tablets or capsules or $100 \mathrm{ml}$ to $500 \mathrm{ml}$ of bottles or a pack of 10 vials or ampoules of injections can be tagged and tracked. This would offer the facility to track products right up to the retail pharmacy level or at least to a transportable shipper or a wholesaler.

\section{F. Dry Diversion Prevention}

Diversion or (Re-channeling) means directing an item of medical supply of low price range (say bulk hospital supplies) to the high price range market sector (retail). Or it can be illegal transport of a traded product from one state to other state where the local tax structure is different and more profitable. Such diversions are nearly impossible to quantify until RFID is in place. RFID enables us to identify and act on diverted products to prevent their reentry in the supply chain $[1,7]$.

\section{G. Counterfeit Drugs}

Tamper-proof RFID tags can also be used for preventing drug counterfeiting. It is assumed that the pharmacies and hospitals across the globe would have a shared secure database, which would be used to verify drugs $[1,7]$.

\section{NOVEL APPLICATIONS OF RFID BASED ON INFORMATION HIDING CONCEPTS}

Information hiding is a science of concealing information. It is an art of communicating in a way that hides the very existence of the communication. Some examples of information hiding include, hiding copyright information in digital images or videos or hiding secret letter in a photograph. This technique to hide information within a digital media is termed as Steganography. Steganography can be used for hiding some crucial information on RFID tags. In this section, we explain how this can be achieved. Initial work in this domain can be found here $[3,4,5,6]$.

\section{A. Expiry Date Hiding}

Steganographic techniques can be used to hide expiry date of pharmaceutical drugs on the RFID tags. This would prevent the sale of expired drugs, e.g. if an expired drug is scanned by the RFID reader, it would flash a signal saying this drug is already expired and should not be sold. However this could also be 
achieved by querying the database, but if the expiry data is hidden on the tag itself, it would enormously reduce the network bandwidth usage. The RFID reader itself can verify if the drug is still good to be sold or not. This can be easily implemented on a RFID tag. RFID tag offers enough space to hide the month and the year of expiry of the drugs. As mentioned earlier, RFID tag is composed of four data structures represented i.e. Header, EM, OC, and SN. The expiry date can be hidden in the Serial number tag, which offers 36 bits of space. To represent month and year (MMYY format), we only need a maximum of 11 bits, which means we have 25 bits for serial number generation. These 25 bits can be used to represent $33,554,432$ unique items belonging to one product, produced by one manufacturer. Hence this serves both the purposes i.e. offer unique identification as well as hiding expiry date on the RFID tag.

\section{B. Tamper Detection}

Another application of information hiding in pharmaceutical industry is tamper detection of RFID tags. RFID tags could be overwritten or tampered by another RFID reader. If the tags are tampered, they could represent wrong drug, when scanned by the RFID reader. This case could become even worse when smart shelves are coupled with RFID technology. In a smart shelf scenario, wrong drugs might be picked up and delivered because the tampered RFID tag could points to a different drug, and this could even result in incorrect diagnosis. The original solution to address data tampering was presented by the authors in $[3,5]$. This solution embeds a secret pattern, generated from the EM and OC, in the serial number SN, in a similar manner as in hiding the expiry date. It is shown in [3, 5], that only 5 to 9 bits would be sufficient to hide the secret pattern for tamper detection.

\section{Fraud Detection and Prevention}

Information hiding and RFID can be used together to detect and prevent RFID frauds. A simple example of RFID fraud is replacing a tag from a cheaper pharmaceutical product to an expensive pharmaceutical product, in an attempt to save cost. For example, replacing the RFID tag from expensive medicines (like those used for curing heart diseases), with simple pain killers. The initial solution to address this is presented in $[3,6]$. The basic idea is to hide the weight of the product in the respective RFID tag. This weight would be checked when the RFID reader scans the RFID tagged product at the point of sale (POS). If a mismatch is detected, it would indicate attempted fraud. This solution could then be used to prevent any further attempts of fraud.

\section{CONCLUSION AND Future WORK}

What we discussed in this paper is only the tip of the iceberg, of the possible applications of RFID technology in pharmaceutical industry. However as the pharmaceutical industry gets more familiar with this technology and as the cost of using this technology also reduces to an affordable level, the scope of application will be ever increasing and in long term the industry will be benefited economically to a large extent. In the future we would like to work more on addressing the tamper detection and information hiding solutions for the pharmaceutical industry. We plan to work closely with the pharmaceutical industry to test our solutions.

\section{REFERENCES}

[1] Sandip Lahiri, "RFID Sourcebook", IBM Press Pearson Plc, New Jersey, USA, 2006.

[2] S. Leaver, "Evaluating RFID Middleware", http://www.forrester.com/Research/Document/Excerpt/0,7211,34390,00. html

[3] Vidyasagar Potdar, Chen Wu, Elizabeth Chang, "Tamper Detection for ubiquitous RFID enabled Supply Chain," In Y. Hao et al. (Eds.): CIS 2005, Part II, LNAI 3802 Springer-Verlag Berlin Heidelberg 2005 and Proceedings of the International Conference on Computational Intelligence and Security (CIS05), pages 273-278, 2005.

[4] Vidyasagar Potdar, Chen $\mathrm{Wu}$, Elizabeth Chang, E-Supply Chain Technologies and Management, chapter "Automated Data Capture Technologies - RFID”. IDEA Group Reference, Hershey, PA, USA. To Appear in March 2007, Accepted July 2006.

[5] Vidyasagar Potdar, Elizabeth Chang, "Tamper Detection in RFID tags using Fragile Watermarking," To Appear in the Proceedings of the IEEE International Conference on Industrial Technology (ICIT06), Mumbai, INDIA, 15-17 Dec, 2006.

[6] Madan Mohan, Vidyasagar Potdar, Elizabeth Chang, "Recovering and Restoring Tampered RFID Data using Steganographic Principles," To Appear in the Proceedings of the IEEE International Conference on Industrial Technology (ICIT06), Mumbai, INDIA, 15-17 Dec, 2006.

[7] Patrick J. Sweeney II, "RFID for Dummies", Wiley Publishing Inc., New Jersey, USA, 2005.

[8] S. A. Weis, S. E. Sarma, R. L. Rivest, D. W. Engels, "Security and Privacy Aspects of Low-cost Radio Frequency Identification Systems", in D. Hutter et al. Edn. Security in Pervasive Computing 2003, LNCS 2802 , pp. 201-212, 2004

[9] R4 Global Solutions Inc. "RFID, Data Synchronization and Standard Data Structures: EAN.UGC, GTN, and EPC", Technical Briefing, Nov 2004

[10] Process Validation. USFDA, www.fda.gov/CDER/GUIDANCE/pv.htm. Accessed on Thursday, November 02, 2006 